\title{
Comparison of human isogeneic Wharton's jelly MSCs and iPSC-derived MSCs reveals differentiation-dependent metabolic responses to IFNG stimulation
}

\author{
Liani Devito ${ }^{1}$, Michail E. Klontzas $\mathbb{1}^{2}$, Aleksandra Cvoro ${ }^{3}$, Antonio Galleu ${ }^{4}$, Marisa Simon ${ }^{3}$, Carl Hobbs ${ }^{5}$, Francesco Dazzi ${ }^{4}$, \\ Athanasios Mantalaris ${ }^{2,6}$, Yacoub Khalaf ${ }^{1}$ and Dusko llic (10 ${ }^{1}$
}

\begin{abstract}
Variability among donors, non-standardized methods for isolation, and characterization contribute to mesenchymal stem/ stromal cell (MSC) heterogeneity. Induced pluripotent stem cell (iPSCs)-derived MSCs would circumvent many of current issues and enable large-scale production of standardized cellular therapy. To explore differences between native MSCs (nMSCs) and iPSC-derived MSCs (iMSCs), we developed isogeneic lines from Wharton's jelly (WJ) from the umbilical cords of two donors (\#12 and \#13) under xeno-free conditions. Next, we reprogrammed them into iPSCs (iPSC12 and iPSC13) and subsequently differentiated them back into iMSCs (iMSC12 and iMSC13) using two different protocols, which we named ARG and TEX. We assessed their differentiation capability, transcriptome, immunomodulatory potential, and interferon- $\gamma$ (IFNG)-induced changes in metabolome. Our data demonstrated that although both differentiation protocols yield iMSCs similar to their parental nMSCs, there are substantial differences. The ARG protocol resulted in iMSCs with a strong immunomodulatory potential and lower plasticity and proliferation rate, whereas the TEX protocol raised iMSCS with a higher proliferation rate, better differentiation potential, though weak immunomodulatory response. Our data suggest that, following a careful selection and screening of donors, nMSCs from umbilical's cord WJ can be easily reprogrammed into iPSCs, providing an unlimited source of material for differentiation into iMSCs. However, the differentiation protocol should be chosen depending on their clinical use.
\end{abstract}

\section{Introduction}

Mesenchymal stromal/stem cells (MSCs) are a heterogenous population of fibroblastic cells that can be isolated from multiple tissues ${ }^{1,2}$. The plasticity, in vitro proliferation, immunomodulatory characteristics, and low immunogenicity of these cells made them an important instrument in the treatment of severe graft vs. host disease $(\mathrm{GvHD})^{3}$. The number of clinical trials involving MSCs

\footnotetext{
Correspondence: Dusko llic (dusko.ilic@kcl.ac.uk)

'Department of Women and Children's Health, King's College London, Guy's Hospital, London, UK

${ }^{2}$ Department of Chemical Engineering, Imperial College London, London, UK Full list of author information is available at the end of the article.

Edited by A. Peschiaroli
}

has been steadily increasing-from 218 clinical trials in 2012 till nearly 900 now. In spite of the rapidly growing body of literature and multiple clinical trials that have demonstrated the therapeutic benefits of MSC-mediated immune modulation variability among donors, nonstandardized methods for their isolation and characterization produced inconsistent results from MSC-based cellular therapies $^{4,5}$. The lack of standardization has been acknowledged by regulatory bodies and calls were made to improve characterization and refine selection criteria for establishing optimal classes of $\mathrm{MSCs}^{6-9}$.

To circumvent these issues, numerous groups, instead of working with primary MSCs, turned to human pluripotent stem cells using both human embryonic and 
induced pluripotent stem cells (hESC/iPSCs) as a source for derivation of MSC-like cells with potent therapeutic properties $^{10-21}$. hESC/iPSC-derived MSCs could be derived under reproducible conditions and easily adapted to large-scale production of standardized cellular therapy. Indeed, quite recently, in May 2017, the first patient with steroid-refractory GvHD has been treated with hiPSCderived MSCs in a clinical trial ongoing in the UK and Australia ${ }^{22,23}$.

However, to the best of our knowledge, only one study reported comparison of primary MSCs and hiPSC-derived MSCs in an isogeneic system-they reprogrammed bone marrow (BM) MSCs into hiPSC and then re-differentiated them back into $\mathrm{MSC}^{24}$. Comparison of DNA methylation between native MSC (nMSC) and hiPSC-derived MSC (iMSC) revealed that although iMSC are similar to nMSC, they do not entirely re-acquire immunomodulatory function in vitro nor do they achieve the original methylation patterns associated with tissue type and ageing.

We explored the isogeneic nMSC/iMSC approach and tested a different source of MSCs, different methods of reprogramming and multiple differentiation protocols. Instead of BM MSCs, we opted for MSCs isolated from Wharton's jelly in umbilical cords (WJ MSC). WJ MSC are easily obtained, isolated, and expanded in vitro under defined xeno-free (dXF) conditions ${ }^{25}$. We also exploited genome non-integrating reprogramming approaches with Sendai virus (SeV)-based delivery instead of using traditional retroviral vector systems ${ }^{26}$. Finally, to differentiate iMSCs from iPSCs, we used two protocols: one described by Frobel et al. ${ }^{24}$ labeled ARG and one based on the experimental approach by Zhao et al. ${ }^{14}$ labeled TEX (Supporting Information Fig. S1).

\section{Methods and materials \\ nMSC derivation and maintenance}

The National Health Service Research Ethics Committee has approved the protocol for isolation of MSCs from perinatal tissues for cellular therapy (14/SW/0042). The collection of umbilical cords, derivation of MSCs, and their maintenance were described previously ${ }^{25}$.

\section{Reprogramming of WJ MSC}

The native MSCs (nMSCs) from two donors, \#12 and $\# 13$, were reprogrammed using CytoTune ${ }^{\circledR}$-iPS Sendai Reprogramming kit. Reprogramming and detection of $\mathrm{SeV}$ expression in iPSC lines was described previously ${ }^{26}$. The modifications include a replacement of animal-based reagents with the xeno-free ones.

\section{Cell culture}

iPSC, nMSC, and iMSC lines were expanded under xeno-free conditions in $5 \% \mathrm{O}_{2}, 5 \% \mathrm{CO}_{2}$ at $37^{\circ} \mathrm{C}$ as described previously ${ }^{25}$. In brief, nMSC lines that were derived and cultured under $\mathrm{dXF}$ conditions, on CELLstart (Thermo Fischer)-coated surface in StemGro ${ }^{\circledR}$ MSC Medium (Corning), were used for reprogramming into iPSC lines. iPSC lines were expanded on Vitronectin XF (StemCell Technologies)-coated surface in TeSR2 (StemCell Technologies). For comparative analyses in this study, nMSC and iMSC lines were propagated in $\alpha$ Minimum Essential Medium ( $\alpha M E M$, Thermo Fisher) supplemented with 10\% human platelet lysate (hPL) (Cook Regentec). To avoid potential effect from different lots of hPL, the same lot has been used for culture of all lines in each of the experiments.

For passaging iPSC lines we used Accutase $^{\mathrm{TM}}$ (STEMCELL Technologies) and for nMSC and iMSC TripLE (Thermo Fisher).

To assess effects of interferon- $\gamma$ (IFNG) on MSCs, recombinant human IFNG (Peprotech) was added to the media at $10 \mathrm{ng} / \mathrm{ml}$ and cells were cultured for $18 \mathrm{~h}$ prior to collection for RNA extraction

Acute myeloid leukemia cell line Katsumi, used as a CD34/CD45-positive control for flow cytometry, was cultured in RPMI-1640 medium supplemented with 20\% fetal calf serum (FCS) at $37^{\circ} \mathrm{C}, 5 \% \mathrm{CO} 2,20 \% \mathrm{O} 2$.

\section{Characterization of iPSC lines}

Characterization of iPSC lines has been described in detail previously ${ }^{26-29}$. Once the lines are confirmed negative for $\mathrm{SeV}^{26,27}$, they are subjected to further characterization:

- Morphology-iPSC lines have to (i) propagate for $>3$ passages, (ii) have well-defined colony edges, (iii) form a tightly packed colony, and (iv) have an absence of differentiated cells.

- Detection of pluripotency-associated markersimmunodetection of pluripotency-associated markers TRA-1-81, POU class 5 homeobox 1 (POU5F1)/octamer-binding transcription factor 4 (OCT4), TRA-1-60, and NANOG ${ }^{27,28}$.

- Determination of genomic stability-we use arraycomparative genomic hybridization to determine genomic stability of the iPSC lines every three months of continuous culture ${ }^{27,28}$.

- Genotyping-amplification of polymorphic microsatellite markers is used for cell line identification at the molecular level ${ }^{27,28}$.

- Differentiation into three germ layers in vitro-iPSC colonies were left to differentiate without passage over a period of at least 3 weeks in Dulbecco's modified Eagle's medium supplemented with 10\% FCS (Hyclone). The cells are then fixed and permeabilized in the dish. Markers representing the three germ layers, mesoderm, ectoderm, and endoderm, are detected with immunostaining ${ }^{27,28}$. 
- Differentiation into three germ layers in vivo-2x $10^{6}$ iPSC suspended in Matrigel were injected subcutaneously into flanks of NOD/SCID mice. The mice were killed 6-10 weeks later, depending on size of the tumor. Tumors are dissected and fixed in 4\% paraformaldehyde. Tumor sections are stained with hematoxylin and eosin and analyze them under a light microscope. The presence of tissue-specific markers of all three germ layers has been confirmed by immunohistochemistry ${ }^{27,28}$.

\section{Characterization of $\mathrm{nMSC}$ and iMSC lines}

Characterization of MSC lines has been described in detail previously ${ }^{25,29}$. In brief:

- Flow cytometry analysis and cell sorting $-1 \times 10^{6}$ cells were resuspended in $100 \mu \mathrm{L}$ of ice-cold phosphate buffer saline (PBS) supplemented with $10 \%$ FBS, mixed with the appropriate primary antibody 1:50 and incubated in the dark for $15 \mathrm{~min}$ at room temperature. After washing with $\mathrm{PBS}$, the cells were resuspended in $0.5 \mathrm{ml}$ of cold PBS supplemented with 10\% FCS. All antibodies used in flow cytometry and cell sorting were purchased from Miltenyi Biotec: phycoerythrin-conjugated antiCD34, anti-CD73, and anti-mesenchymal stromal cell antigen-1 (MSCA-1), allophycocyaninconjugated anti- CD56, anti-CD29 and anti-CD105, fluorescein-conjugated anti-CD44, anti-CD90, and anti-CD271, and Vio-Blue-conjugated anti-CD45. Titration was done for each of antibody used. Unstained cells were used as negative control. Fluorescence minus one was also performed to avoid any nonspecific binding. Expression of cell surface molecules was assessed using a FACS CANTO II (BD) and the data were analyzed using the DIVA software.

- Differentiation assays-the differentiation potential of nMSCs and iMSCs has been validated in three independent experiments using complete STEMPRO $^{\circledast}$ adipogenesis, chondrogenesis or osteogenesis differentiating medium (Gibco ${ }^{\circledR}$, Life Technologies) according to manufacturer's protocol. Following fixation, the cellular lipids were detected cells with LipidTOX ${ }^{\mathrm{TM}}$ Green Neutral Lipid Stain (Thermo Fisher) and nuclei with Hoechst 33342 (Thermo Fisher). The samples were mounted in Vectashield (Vector) and visualized using epifluorescence microscope (Nikon ECLIPSE 50i). $\mathrm{Ca}^{2+}$-containing cells in osteogenic cultures were stained with Alizarin red, whereas the presence of cartilage-related glycosaminoglycans in chondrogenic cultures was detected with Alcian blue staining.

- Real-time quantitative PCR (qPCR)-mRNA expression levels of osteogenic (RUNX2), chondrogenic (COL11A1), and adipogenic (FABP4) markers in all nMSC and iMSC lines were determined using the iScript cDNA Synthesis Kit (Bio-Rad) and SYBR Green Mastermix (Roche) in LightCycler 480 II (Roche). The primer sequences are listed in Supporting Information (Table S1).

\section{Differentiation of iPSC into iMSC}

We generated iPSC-derived MSCs (iMSCs) using xenofree modifications of two protocols. The first differentiation protocol, called ARG, was based on the protocol described by several groups ${ }^{13,24,30}$ and lasted 30 days. The iPSCs disaggregated into single cell suspension were seeded on Vitronectin XF-coated plates and cultured in aMEM (Thermo Fisher) supplemented with 10\% hPL (Cook Regentec). Until Day 14 of differentiation, the medium also contained 2\% B27 Supplement (Thermo Fisher) and the passaging was done in the presence of $10 \mu \mathrm{m}$ Y27632 (STEMCELL Technologies).

The second protocol, called TEX, was based on the experimental approach by Zhao et al. ${ }^{14}$, who used SMAD $2 / 3$ inhibitor to promote differentiation. In brief, the cells were plated on Vitroncetin XF-coated wells of six-well plates and cultured in TeSR2 supplemented with $10 \mu \mathrm{M}$ SB-431542 (Sigma) for 25 days with continuous passaging (every 3-4 days) under the same culture conditions. During the next 20 days the cells were passaged on uncoated plastic. And the culture medium was then switched to standard MSC medium ( $\alpha$ MEM supplemented with $10 \% \mathrm{hPL}$ ). All inhibitors were dissolved according to manufacturer's recommendation.

At the end of the differentiation protocols, cells that were triple positive for $\mathrm{CD} 73, \mathrm{CD} 90$ and $\mathrm{CD} 105$ were sorted with a FACSAria II cell sorter (BD) and FACSDIVA software (BD) for acquisition and analysis, expanded and used for subsequent experiments.

\section{Transcriptomics}

Total RNA was isolated from cells using the QIAgen RNeasy Mini kit (Qiagen). The RNA quality was assessed using Agilent RNA 6000 Nano Kit and the 2100 Bioanalyser (Agilent).

For the HumanHT-12 v4 Expression BeadChip (Illumina) assay RNA was isolated from cells with and without $18 \mathrm{~h}$ IFNG treatment. The samples were prepared using SuperScript III Reverse Transcriptase (Thermo Fisher) and TargetAmp Nano Labeling kit (Lucigen). Hybridization was performed according to the manufacturer's instructions and run on the iScan system (Illumina). The data were analyzed by GenoSplice Technology using algorithm EASANA 2015.1.

The expression of the IFNG-target genes indoleamine 2,3-dioxygenase 1 (IDO1), guanylate-binding protein 4 (GBP4) and C-X-C motif chemokine ligand 11 (CXCL11) 
was assessed using the same RNA that was used for the BeadChip essay. Quantitative reverse transcription polymerase chain reaction (RT-qPCR) was performed using the StepOne System Instrument (Thermo Fisher) using a TaqMan $^{\circledast}$ RNA-to-CT ${ }^{\mathrm{ma}}$ 1-Step Kit (Thermo Fisher) according to the manufacturer's instructions. Data were collected and analyzed using the comparative threshold cycle method with hypoxanthine phosphoribosyltransferase 1 (HPRT1) as the reference gene. The primers are listed in Supporting Information (Table S1).

\section{Metabolomics}

Metabolomics analysis was performed as previously reported $^{31-33}$. In brief, culture medium was aspirated, cells were washed with PBS and metabolism quenched with icecold $\left(-20^{\circ} \mathrm{C}\right)$ methanol. Following the addition of highperformance liquid chromatography grade water (1:1 methanol:water), ribitol ( $1 \mathrm{mg} / 10^{6}$ cells, Sigma-Aldrich) and ${ }^{13} \mathrm{C}$-glucose $\left(2 \mathrm{mg} / 10^{6}\right.$ cells, Alfa Aesar) were used as internal standards based on the results of DNA quantification $^{34}$. Samples were dried overnight under vacuum and the resulting dry polar metabolite mixtures were derivatized with the addition of $50 \mu \mathrm{L}$ of methoxyamine hydrochloride $(20 \mathrm{mg} / \mathrm{mL}$ in pyridine, Sigma-Aldrich) for $1.5 \mathrm{~h}$, followed by $100 \mu \mathrm{L}$ of $N$-methyl-trimethylsylyltrifluoroacetamide for $6 \mathrm{~h}$ (Alfa Aesar) ${ }^{35}$. Each sample was run in quadruplicate injections in a Shimadzu QP2010 Ultra gas chromatography-mass spectrometry (GC-MS, Shimadzu).

\section{Statistics}

qPCR data for markers of adipo-, chondro-, and osteogenesis were collected and analyzed using the comparative threshold cycle method with TATA-box binding protein $(T B P)$ and glucuronidase $\beta(G U S B)$ as reference genes. The mean \pm standard deviation (SD) was calculated $(n=3$ of each line), and statistical analysis was performed using the Prism curve-fitting program (GraphPad Prism, version 6.01). $P$ values for expression of IFNG-target genes were determined with t-test and adjusted using Bonferroni correction. $p \leq 0.05$ indicates statistically significant and $p \leq 0.001$ highly significant values.

Transcriptomics data were analyzed by GenoSplice Technology using algorithm EASANA 2015.1. We used the R/Bioconductor software package limma that provides an integrated solution for differential expression analyses of data from gene expression experiments. To determine whether iMSC12 ARG or iMSC12 TEX is closer to paternal nMSC12, we used the R/Bioconductor software packages limma and hclust for Euclidean distance.

Metabolomics data preparation was accomplished according to Kanani et al. ${ }^{36}$ and bioinformatics analysis was performed with the use of $\mathrm{R}$ ( $\mathrm{R}$ version 3.4.2, $\mathrm{R}$ studio 1.1.442) and TM4 MeV (version 4.9). Principal component analysis and hierarchical clustering with Manhattan distance metric were used for exploratory unsupervised multivariate analysis. Statistical analysis of multidimensional metabolomics data was performed with the use of Significance Analysis for Microarrays ${ }^{37}$ with the minimum significance threshold $(\delta)$ yielding an false discovery rate-median of $0 \%$. Significant metabolites for each comparison were depicted on a reconstruction of the core MSCs' metabolic network.

\section{Results \\ Reprogramming and characterization of iPSC lines}

We have previously demonstrated that WJ MSCs could be isolated successfully from the umbilical cord under $\mathrm{dXF}$ conditions ${ }^{25}$. The cells derived in this way retain all the characteristics of MSCs: the expression of typical MSC surface "markers", differentiation potential into adipocytes, chondrocytes and osteocytes, and immunomodulatory activity. Therefore, to test our hypothesis that clinical grade iPSC-derived WJ MSCs can be generated from the beginning to the end under xeno-free conditions, we chose WJ MSCs derived and propagated under dXF conditions from two of seven donors for reprogramming into iPSC lines. The criterion for the selection of the donors was the size of the MSCA-1 + subpopulation (Supporting Information Table S2). MSCA-1 + cells are mesenchymal precursor cells and their expression is linked with an increased capacity of colony forming units $>100$-fold in comparison with the MSCA-1- subpopulation $^{38-40}$. WJ MSCs derived under dXF that had the highest number of MSCA-1 + cells were from donor 13 (86.1\%), 12 (38.8\%), and 14 (29.5\%). To distinguish them from iPSC-derived MSC lines, we dubbed the primary native WJ MSCs as nMSCs. Therefore, from donor 12, we have $\mathrm{nMSC} 12$ and from donor 13, we have nMSC13. Both lines were from female donors (Supporting Information Fig. S2).

Following reprogramming, one colony from each background was expanded further and adapted to feederfree conditions (Fig. 1a) ${ }^{41}$. SeV was undetectable after 60 days in culture (Fig. 1b) and we could proceed with the characterization of the iPSC12 and iPSC13 lines. Both lines express pluripotency-associated markers, TRA-1-81, POU5F1 (OCT4), TRA-1-60, and NANOG (Fig. 1c) and can differentiate spontaneously into cells/tissues from all three germ layers in vitro (Fig. 1d) and in vivo (Fig. 1e).

\section{Differentiation of iMSCs and their characterization highlighted diversity of the MSC populations}

To determine how close MSCs differentiated from pluripotent stem cells are to nMSC, we followed two different protocols: $\mathrm{ARG}^{13,24,30}$ and $\mathrm{TEX}^{14}$ with slight modifications to make them xeno-free. A difference between the two protocols was noted in cell morphology 


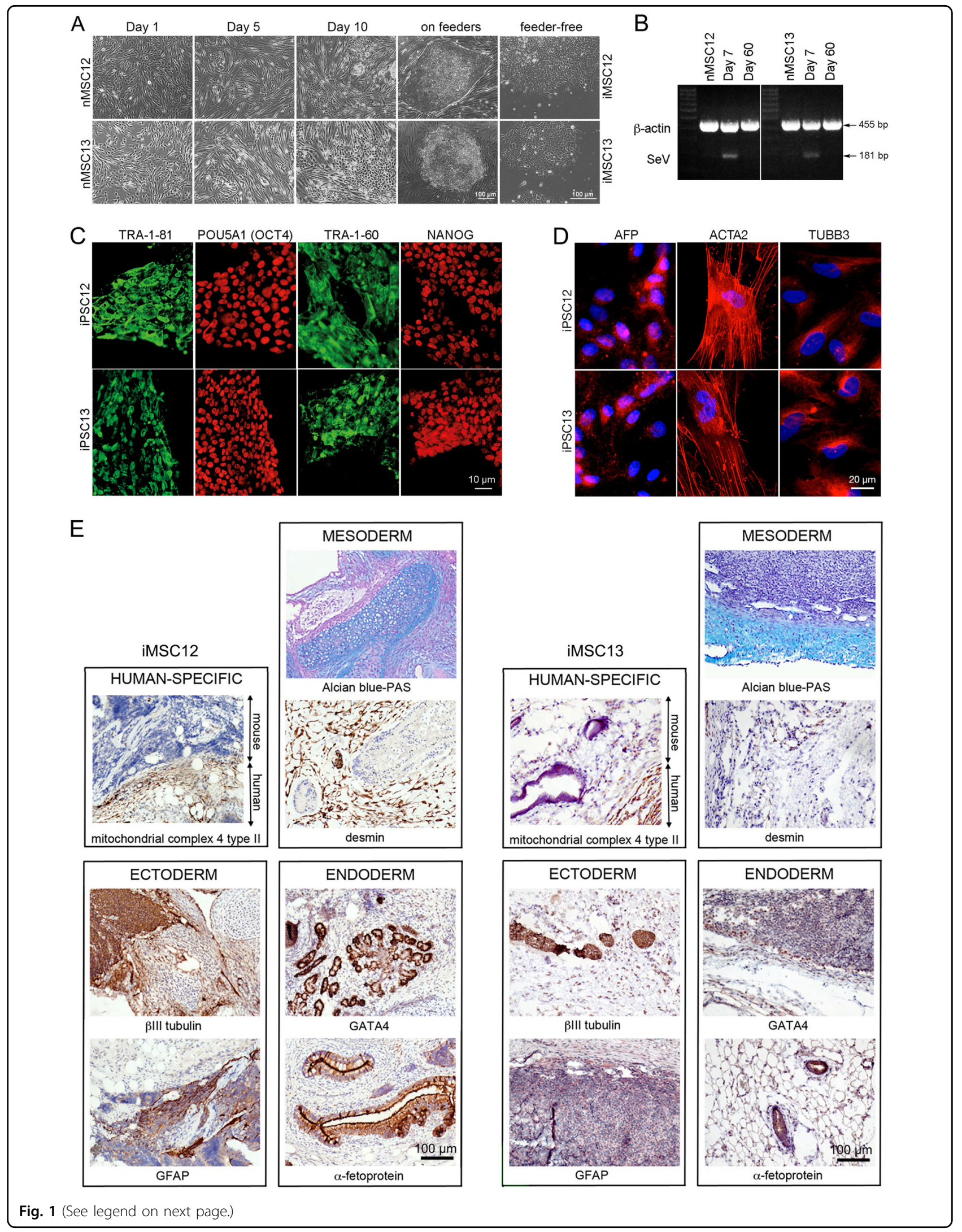


(see figure on previous page)

Fig. 1 Reprograming nMSC into iPSC and their characterization. a Changes in morphology of nMSC12 during SeV-mediated reprogramming. b SeV was undetectable in iMSC12 and iMSC13 2 months after transduction. Original non-transduced native MSC lines (nMSC12 and nMSC13) were used as negative controls; 7 days post SeV transduction cells were used as positive controls (Day 7). At Day 60 in culture, the cells were negative for SeV expression. B-actin (ACTB) PCR product is $455 \mathrm{bp}$, whereas SeV is $181 \mathrm{bp}$. MW: 100 bp ladder. c Both iPSC12 and iPSC13 express pluripotencyassociated markers. iPSC colonies were positive for keratan sulfate antigens TRA-1-60 and TRA-1-81 (green) and transcription factors POU5A1 (OCT4) and NANOG (red). $\mathbf{d}$ iPSC12 and iPSC13 can spontaneously differentiate into derivatives of all three germ layers in vitro. Alpha-fetoprotein (AFP) demonstrated presence of endodermal derivatives, smooth muscle actin alpha 2 (ACTA2) of mesoderm and tubulin beta 3 class III (TUBB3) of ectoderm. e Spontaneous differentiation of iPSC12 and iPSC13 into all three germ layers in vivo is confirmed by detection of specific markers. All sections were stained with hematoxylin and eosin (H\&E), whereas specific stains are brown (immunohistochemistry) or light blue (Alcian). Positive staining for mitochondrial complex IV type II confirms the human origin of the teratoma tissue. Germ layer markers: Alcian blue/periodic acid Schiff (PAS)-stained cartilage and DESMIN for mesoderm, TUBB3 and glial fibrillary acidic protein (GFAP) for ectoderm, and GATA-binding protein 4 (GATA4) and AFP for endoderm

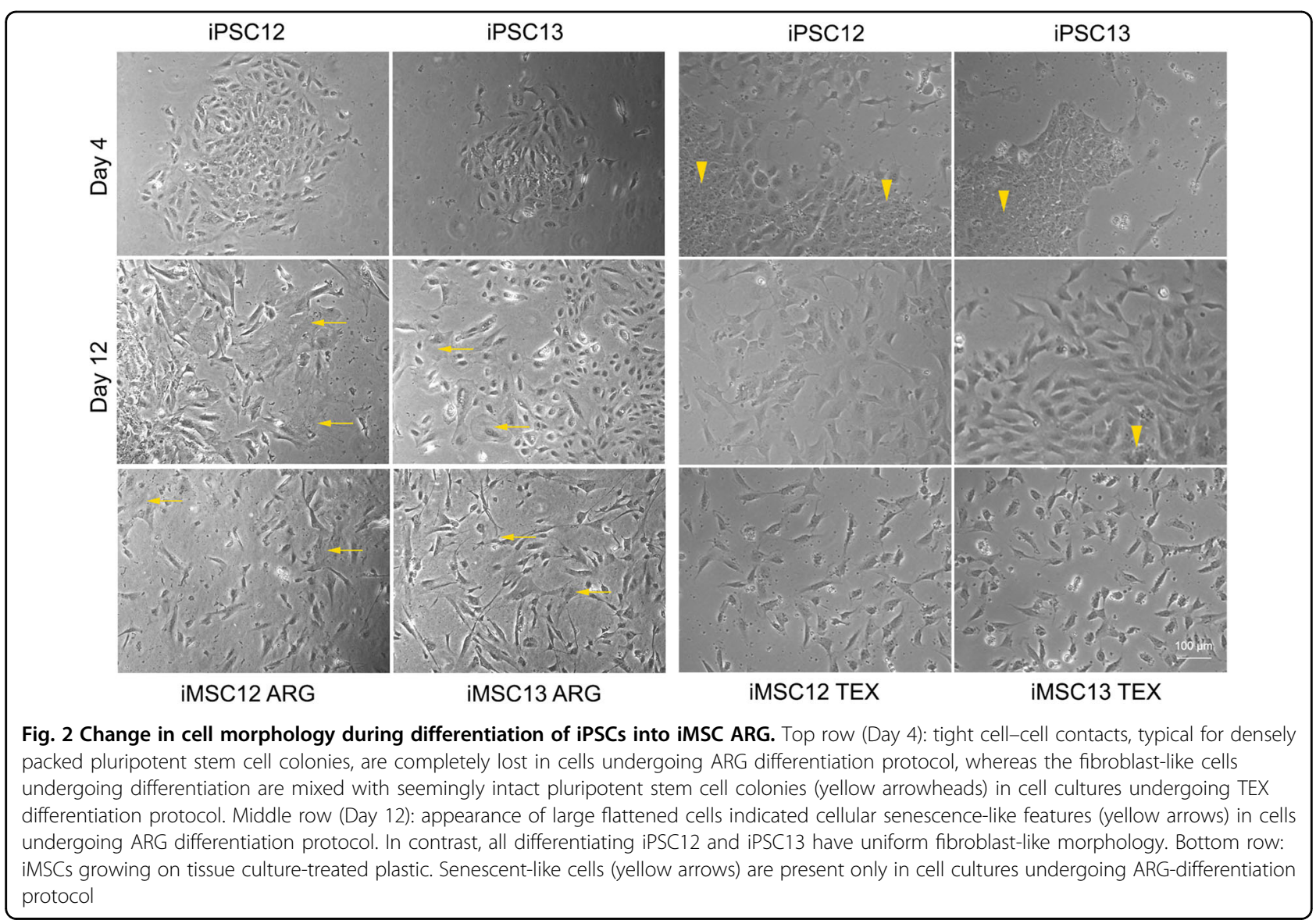

after 4 days (Fig. 2) and it persisted among CD73 + CD90 + CD105 + sorted cells cultured on tissue culture-treated plastic throughout the entire experimental work. Senescent-like cells were observed in both iPSC12 and 13 lines undergoing the ARG differentiation protocol as early as Day 12. They were not observed in either of two lines undergoing the TEX-differentiation protocol. This suggested that the iMSC12 TEX and iMSC13 TEX might maintain some of their stem cell-like characteristics and represent a type of MSC progenitor, whereas iMSC12 ARG, and iMSC13 ARG might be closer to primary MSC, which are a mixture of progenitors and the cells that lost stem cell-like characteristics and have more limited proliferation capacity.

Indeed, following iMSC differentiation into adipo-, chondro-, and osteogenic lineages, we found that the cells generated from iMSC using the TEX protocol had significantly higher expression of adipogenic and chondrogenic markers than those using ARG (Fig. 3). No significant difference between two protocols was observed only after culture under osteogenic conditions. These data are consistent with cellular morphology (Fig. 2), thus suggesting that lines of TEX origin might resemble progenitors more closely. 


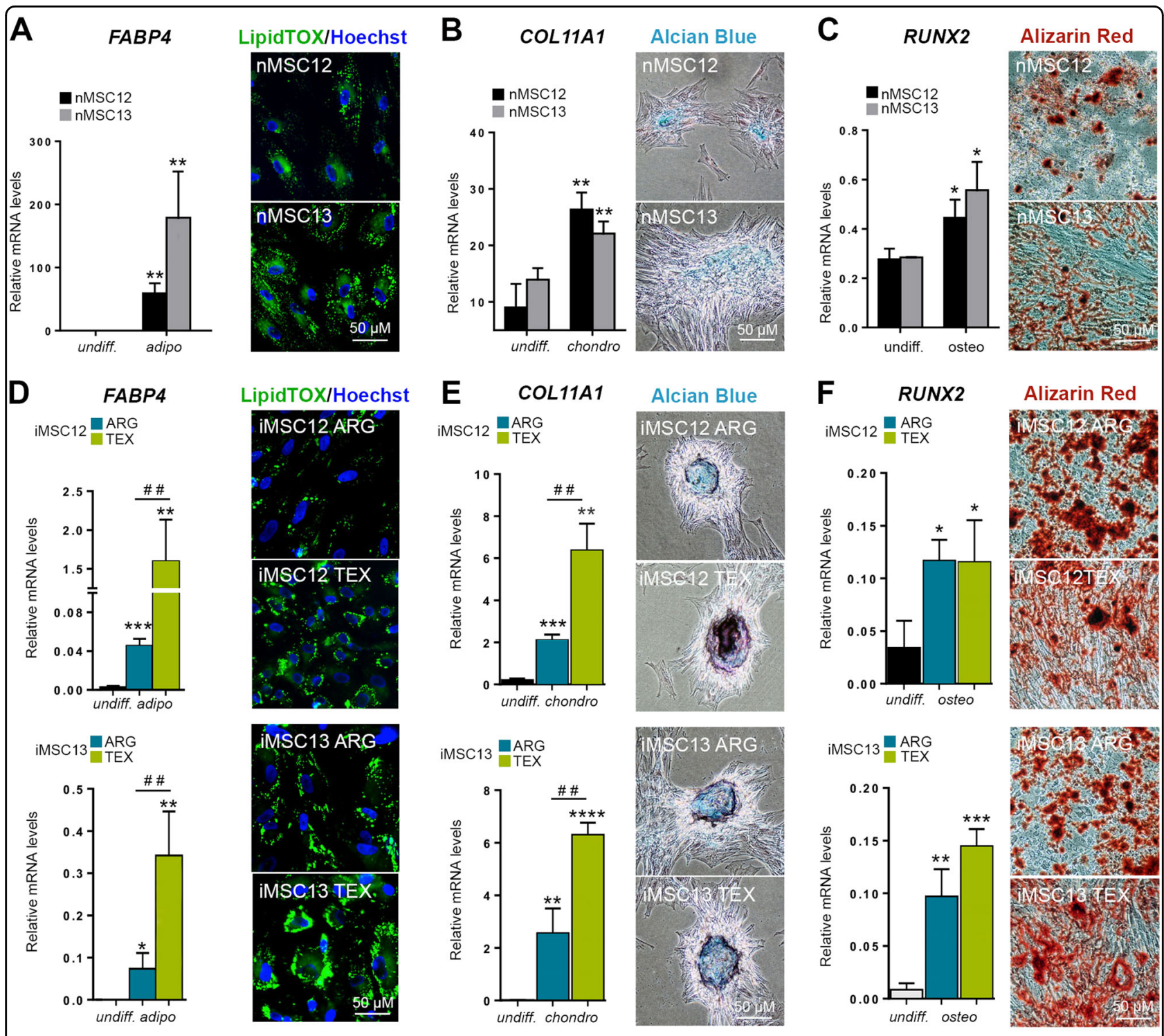

Fig. 3 Differentiation potential of iMSCs. Differentiation potential. a nMSCs that were exposed to adipogenic differentiation medium showed accumulation lipid droplets as detected with LipidTOX stain and had a significantly higher $\left({ }^{* *} p \leq 0.01\right)$ expression of fatty acid binding protein 4 (FABP4) mRNA. No significant difference in FABP4 mRNA levels was detected between nMSC12 and nMSC13. $\mathbf{b}$ nMSCs that were exposed to chondrogenic differentiation medium were positive for cartilage glycosaminoglycans as detected by Alcian Blue staining and had a significantly higher $\left({ }^{* *} p \leq 0.01\right)$ expression of collagen 11 (COL11A1) mRNA. No significant difference in COL11A1 levels was detected between nMSC12 and nMSC13. c nMSCs that were exposed to osteogenic differentiation medium were full of mineral accumulations as detected by Alizarin Red staining and had a significantly higher $\left({ }^{*} p \leq 0.05\right.$ ) expression of runt-related transcription factor 2 (RUNX2) mRNA. No significant difference in RUNX2 levels was detected between nMSC12 and nMSC13. d Following adipogenic differentiation, all iMSCs lines showed accumulation of lipid droplets and significantly higher $\left({ }^{* * *} p \leq 0.001,{ }^{* *} p \leq 0.01,{ }^{*} p \leq 0.05\right.$ ) expression of FABP4. Both lines generated using the TEX protocol (iMSC12 TEX and iMSC13 TEX) had a significantly higher (\#\# $p$ 0.01) expression of FAPB4 than the lines generated using the ARG protocol (iMSC12 ARG and iMSC13 ARG). e Following chondrogenic differentiation, all iMSC lines were positive for cartilage glycosaminoglycans as detected by Alcian Blue staining and had a significantly higher $\left({ }^{* * * *} p \leq 0.0001,{ }^{* *} p \leq 0.001,{ }^{* *} p \leq 0.01\right.$ ) expression of COL11A1. Both lines generated using the TEX protocol (iMSC12 TEX and iMSC13 TEX) had a significantly higher (\#\# $\mathrm{*} \leq .01$ ) expression of COL11A1 than the lines generated using the ARG protocol (iMSC12 ARG and iMSC13 ARG). $\mathbf{f}$ Following osteogenic differentiation, all iMSC lines were full of mineral accumulation and had a significantly higher $\left(^{* * *} p \leq 0.001,{ }^{* *} p \leq 0.01\right.$, ${ }^{*} p \leq 0.05$ ) expression of RUNX2. There were no significant differences in RUNX2 expression between the lines generated using the TEX protocol (iMSC12 TEX and iMSC13 TEX) and the lines generated using the ARG protocol (iMSC12 ARG and iMSC13 ARG) after culture under osteogenic conditions 


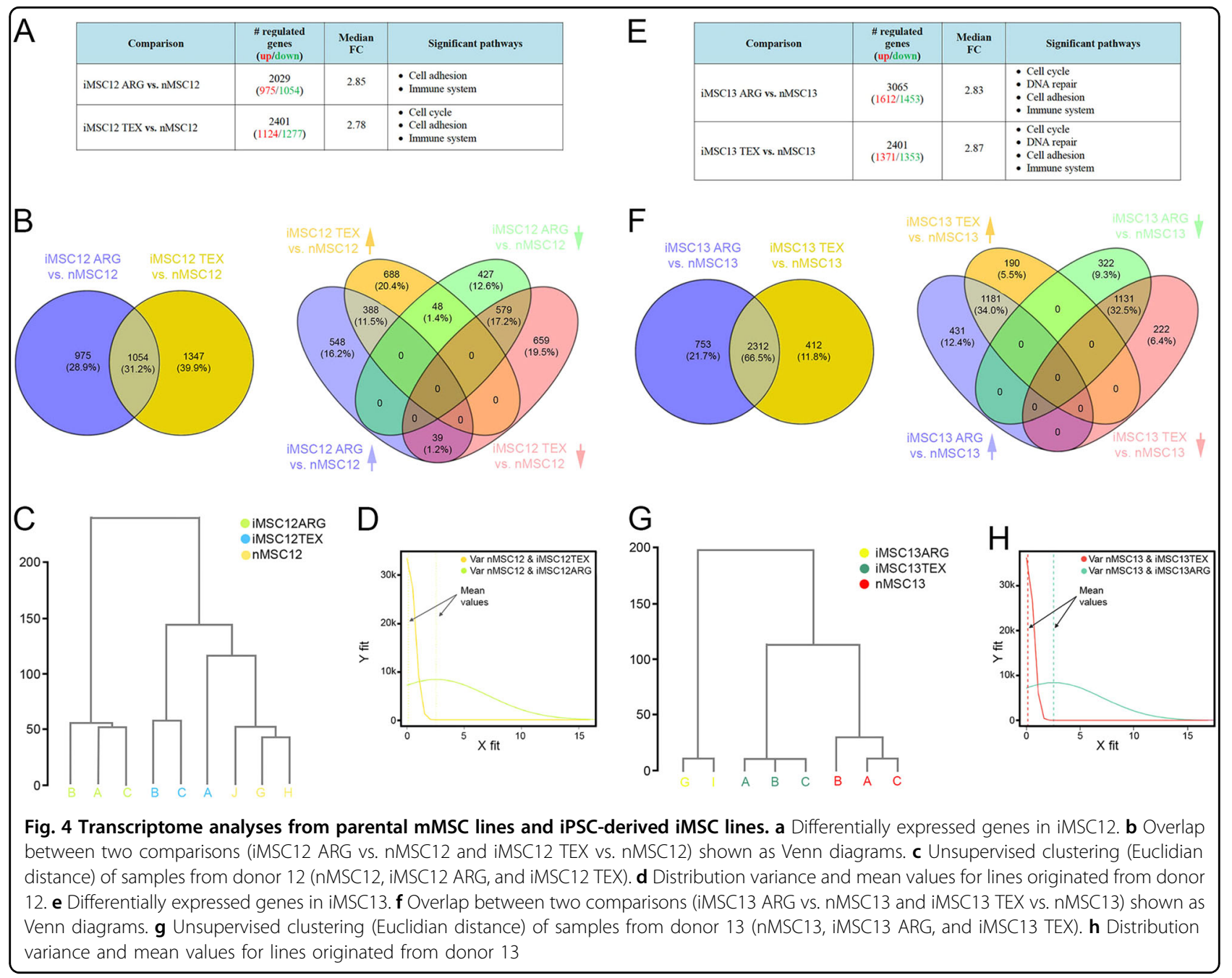

TEX protocol yields iMSC with a transcriptomics profile closer to nMSC than ARG protocol

Next, we compared transcriptome of iMSC12 and iMSC13 with their respective parental lines nMSC12 and nMSC13 (Fig. 4).

When compared with parental nMSC12, the number of differentially expressed genes is higher using TEX than the ARG protocol (2401 in iMSC12 TEX vs. 2029 in iMSC 12ARG; Fig. 4a). However, there were no significant differences between protocols in terms of fold-change median values (2.85 in iMSC12 ARG vs. nMSC12 and 2.78 in iMSC12 TEX and nMSC12). Approximately half of the genes with different regulation in iMSC12 ARG (1054 out of 2029 genes or 51.9\%) are also differently regulated in iMSC12 TEX (1054 out of 2401 genes or 43.9\%). Most of the common genes have the same up-/ downregulation (Fig. 4b). To determine whether iMSC12 ARG or iMSC12 TEX are closer to paternal nMSC12, we used the R/Bioconductor software packages limma and hclust for Euclidean distance. Although there were more- regulated genes with TEX than ARG (2401 vs. 2029), based on the expression of all expressed genes, samples from the TEX protocol (iMSC12 TEX) were closer to nMSC12 than samples from the ARG protocol (iMSC12 ARG) (Fig. 4c). To explain this difference, we checked the variance distribution between iMSC12 ARG/nMSC12 and iMSC12 TEX/nMSC12 (Fig. 4d). The variances were higher between gene expression from the iMSC12 ARG/ nMSC12 groups than between gene expression from the iMSC12 TEX/nMSC12 groups. Unsupervised clustering and variance analysis showed also that iMSC12 TEX cells are closer to nMSC12 than iMSC12 ARG.

Using the same approach, we then determined the differentially expressed genes and their associated pathways between iMSC13 TEX and nMSC13 and between iMSC13 ARG and nMSC13. We found that iMSC13 from both protocols (TEX and ARG) have a high number of differentially expressed genes (Fig. 4e). However, in this case, the number of differentially expressed genes was higher using ARG than TEX (3065 vs. 2724). Similarly, there 
were no significant differences between protocols in terms of fold-change median values (2.83 in iMSC13 ARG vs. nMSC13 and 2.87 in iMSC13 TEX and nMSC13). The majority of genes with different regulation in iMSC13 TEX (2312 out of 2724 genes or $84.9 \%$ ) are also differently regulated in iMSC13 ARG (2312 out of 3065 genes or $75.4 \%)$. Most of the common differently regulated genes have the same up-/downregulation (Fig. 4f). Based on the expression of all expressed genes, samples from the TEX protocol (iMSC13 TEX) were again closer to nMSC13 than samples from the ARG protocol (iMSC13 ARG) (Fig. 4g). The variances were higher between gene expression from the iMSC13 ARG/nMSC13 groups than between gene expression from the iMSC13 TEX/nMSC13 groups (Fig. 4h). Unsupervised clustering and variance analysis together show that iMSC13 TEX cells are closer to $\mathrm{nMSC} 13$ than iMSC13 ARG.

The sum of the data indicates that the TEX protocol produces iMSCs closer to nMSCs.

\section{ARG protocol yields iMSCs that respond to IFNG similar to nMSC}

As MSCs can exert potent immunosuppressive and immunoregulatory effects ${ }^{5,42,43}$, we assessed their response to IFNG treatment $(\mathrm{T})$. To verify that IFNG treatment induced the expected signaling response, we compared mRNA levels of IDO1, GBP4, and CXCL11 between untreated and treated cells by qPCR. These three genes have been used extensively as markers of IFNG response ${ }^{44-46}$. Both nMSC and all iMSC lines displayed a similar increase in expression of these three markers (Fig. 5a).

Next, we performed metabolomics analysis of nMSCs and iMSCs to evaluate the changes in metabolic physiology in response to IFNG treatment and identify potential protocol-specific (ARG or TEX) differences. Treatment with IFNG was found to globally increase the metabolic activity of both nMSCs and iMSC ARG as shown by principal component analysis (Fig. 5b) and hierarchical clustering, where the profiles of iMSC ARG (T) cluster together with the profiles of $\mathrm{nMSC}(\mathrm{T})$ despite the variability in metabolic physiology before IFNG treatment (Fig. 5c). Clustering between iMSC ARG(T) and $\mathrm{nMSC}(\mathrm{T})$ denotes the higher metabolic similarity upon IFNG stimulation between cells derived with ARG and native cells.

Direct on-network significance comparison of nMSCs vs. iMSC ARG, showed that iMSC ARG had a lower glycolytic and glutaminolytic activity, lower levels of tricarboxylic acid cycle (TCA) and urea cycle metabolites and decreased amino-acid pools compared with their native equivalents (Fig. 6a). Upon IFNG stimulation, the differences in metabolic phenotype between nMSC (T) and iMSC ARG (T) were limited to increased levels of glutamine and serine in iMSC ARG (T) (Fig. 6b). iMSC
TEX did not respond to IFN treatment in a similar way to iMSC ARG. iMSC TEX were found to possess a highly variable metabolic physiology. In iMSC TEX (T), glutaminolysis and the levels of biosynthetic precursors (amino acids and lipid precursors such as myoinositol) were significantly lower compared to both other groups (Fig. 6c).

\section{Discussion}

We used a model system of isogeneic primary WJ nMSCs and iPSC-derived iMSCs to explore differences between native WJ MSCs, nMSCs, and iMSCs, originating from these nMSCs. A similar system has been reported by Frobel et al. ${ }^{24}$. They found differences in epigenetic signature, particularly in patterns associated with tissue type and ageing. They also reported incomplete reacquisition of immunomodulatory properties, which prompted us to analyze response to the well characterized proinflammatory cytokine IFNG, secreted by cells of both the innate and adaptive immune systems.

We employed two different differentiation protocols: (1) ARG was very similar to the protocol described by Frobel et al. ${ }^{24}$ and (2) TEX was similar to Zhao et al. ${ }^{14}$. We found that two iMSC ARG lines we analyzed responded similarly to IFNG treatment as parental nMSC, indicating that their immunomodulatory properties might be preserved. There are several differences between our isogeneic system and the system reported by Frobel et $\mathrm{al}^{24}$, which might contribute to the different findings. Interestingly, the data from iMSC lines differentiated using TEX, had attenuated response to IFNG stimulation. We do not know for sure why TEX retained more stem cell-like phenotype improved the differentiation potential, whereas ARG preserved immunomodulation better. We can only speculate that is due to exposure of cells to transforming growth factor beta (TGFB) signaling inhibitor $(10 \mu \mathrm{M}$ SB431542) in TEX protocol. TGFB family signaling is implicated in regulation of stemness, however, the effects are diverse and depend on the cell types as well as cell physiology and environmental factors ${ }^{47}$.

The concept that multiple connective tissues have common progenitor stem cell retained throughout adulthood at various locations in the body has been debated within the community, though not proven yet, and the concept remains ambiguous ${ }^{9}$. BM MSCs that were used for reprogramming and derivation of iMSC are isolated from adults and from a different tissue type then ours. Although WJ MSCs share common features with BM MSCs and MSC from other adult tissues, such as being positive for CD44, CD73, CD90, CD105, $\alpha$-smooth muscle actin, and vimen$\operatorname{tin}^{48,49}$, they still might be a distinct population. Moreover, the term WJ MSCs is often extended to all umbilical cord stromal cells even though they are residing in different distinct zones of the WJ matrix-the sub-amnion, WJ per se, and peri-vascular zone ${ }^{50}$. 


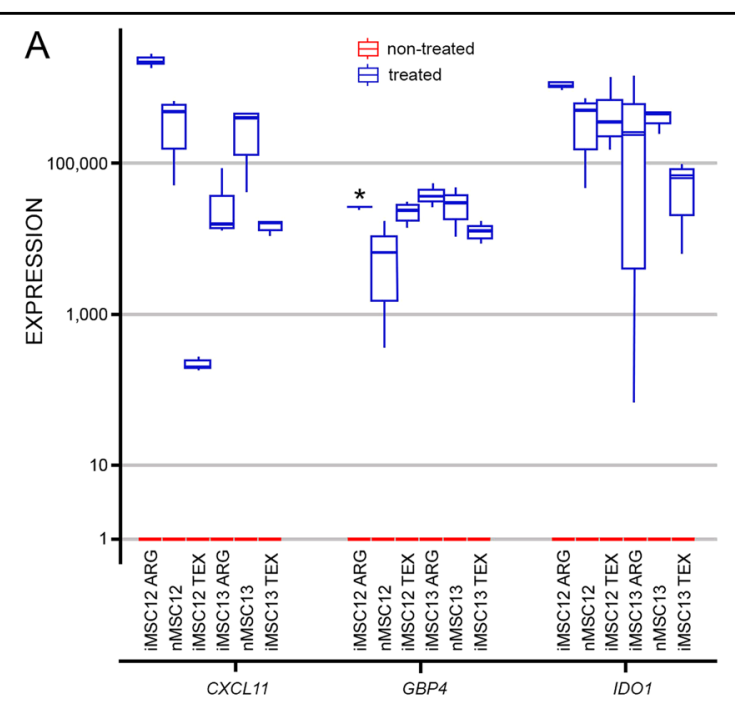

C
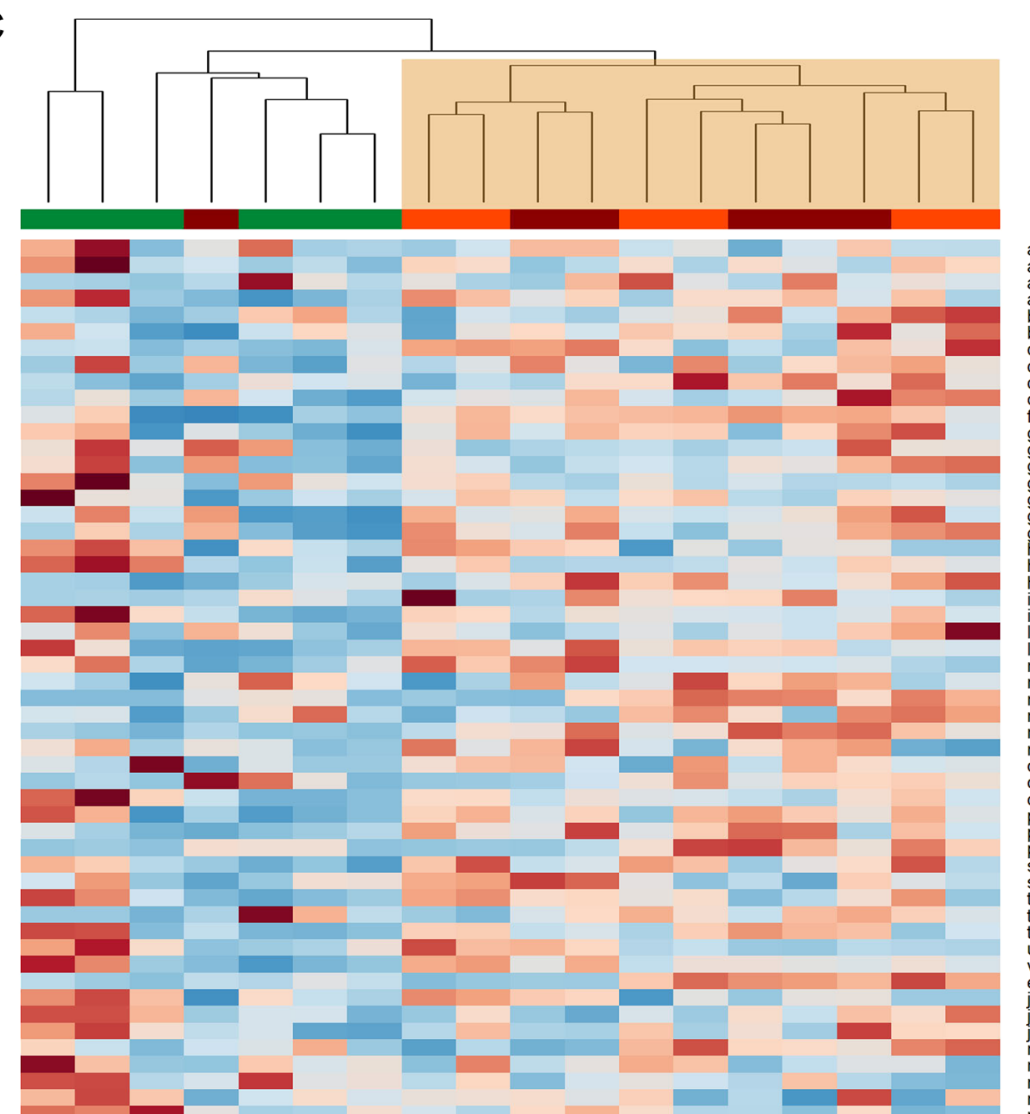

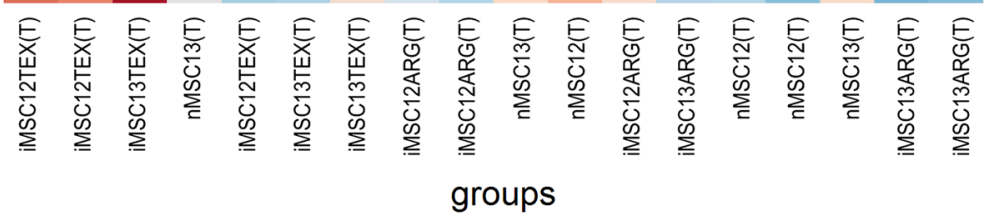

Fig. 5 Different metabolomic response of iMSC to IFNG treatment. a QPCR analysis confirmed that IFNG treatment induced expression of CXCL11, GBP4, and IDOI in all our six iMSC lines ( $n=3$ for each of non-treated and IFNG-treated samples). Red lines represent values in non-treated cells. Only two treated individual lines significantly differed in one gene expression. p values were determined with $t$ test and adjusted using Bonferroni correction. *, $p \leq 0.05$. b, c Upon IFNG treatment (T), PCA (B) and hierarchical clustering with Manhattan distance metric (C) of metabolomics showed increased global metabolic activity only in nMSC (T) and iMSC ARG (T), but not in iMSC TEX (T) lines 


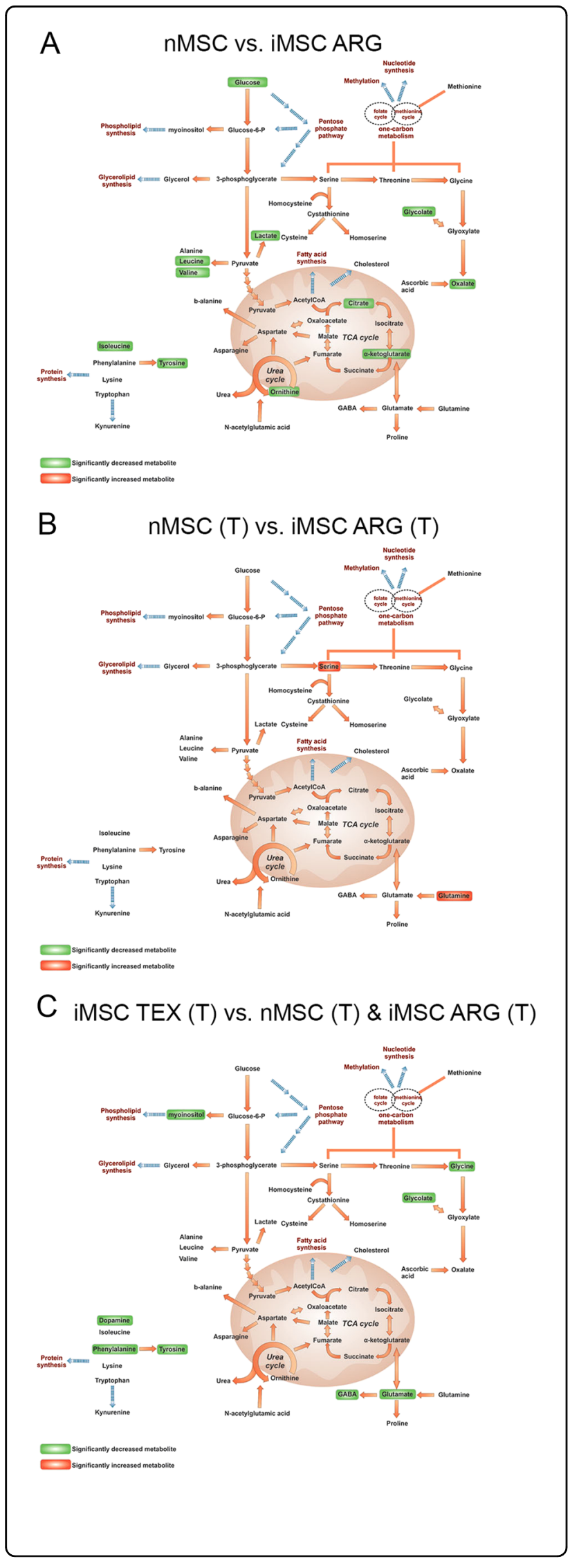

Fig. 6 On-network representation of significant metabolic changes. a Comparison of nMSCs vs. IMSC ARG, showed that iMSC ARG had a lower glycolytic and glutaminolytic activity, lower levels of TCA and urea cycle metabolites and decreased amino-acid pools compared with nMSCs. b IFNG stimulation diminished the differences in metabolic phenotype between nMSC ( $T$ ) and IMSC ARG (T) seen in a. The only difference are increased levels of glutamine and serine in iMSC ARG (T). c INFG treatment did not increase metabolic activity of iMSC TEX (T). Glutaminolysis and the levels of biosynthetic precursors (amino acids and lipid precursors such as myoinositol) was significantly lower in comparison with both nMSC (T) and iMSC ARG (T). (T), IFNG-treated cells

Defects in immunomodulatory response of iMSCs found by Frobel et al. ${ }^{24}$ could be also an effect of difference in culture conditions. Most cells in culture are grown in an atmosphere of $5 \% \mathrm{CO}_{2}, 95 \%$ air, which represents an incubator oxygen concentration of $20 \%(160 \mathrm{~mm} \mathrm{Hg})$. Such tissue culture conditions are strongly oxidizing. For example, oxygen gradients within the human $\mathrm{BM}$ are in a range from below $1 \%$ in hypoxic niches to $6 \%$ in the sinusoidal cavity $^{51}$. It has been demonstrated that such low oxygen levels are essential for maintenance of cell's stemnessrelated properties in all MSCs including adipose-derived ${ }^{52}$ and WJ MSC ${ }^{25,53-56}$. To mimic physiological conditions as much as possible in vitro, we cultured the cells in all stages at $5 \% \mathrm{O}_{2}$. In addition, following modification of the original protocol $^{24,30}$ introduced by Luzzani et al. ${ }^{13}$, we also supplemented the iPSC-differentiation medium with B27 Supplement, which supports cell survival with a defined spectrum of biological antioxidants ${ }^{57,58}$.

Although we used different reprogramming techniques (genome-integrating retrovirus vs. genome nonintegrating $\mathrm{SeV}$ ), we do not believe that they are behind differences in immunomodulatory effects we observed, and those published by Frobel et al. ${ }^{24}$. Three recent studies have compared the genomic features of iPSCs from between 100 and 300 individuals, and the iPSC lines have shown to be heterogeneous, raising questions about the suitability of these lines for genetic studies ${ }^{59-61}$. These studies were able to show that the genetic background of each individual has a superior influence on variation in the iPSC lines compared with other non-genetic factors, including copy number status, culture conditions, passage, and gender. Therefore, it is hypothesized that the majority of iPSC heterogeneity is driven by inherent genetic variation between individuals, rather than by any variation in culture conditions and duration, or in the reprogramming method used. All three reports showed that a great amount of genomic variations between the iPSC lines affected the genes involved in stem cell maintenance and the differentiation efficiency of the iPSCs. Our iMSC lines from both groups, TEX and ARG, showed individual differences in gene expression that could be attributed to the genetic background to each parental cell. 
Stimulation of nMSCs with IFNG lead to increased metabolism (Supporting Information Fig. S3) and diminished metabolic differences between nMSC and iMSC ARG (Fig. 6a, b). However, we could not detect such change in overall metabolism of iMSC TEX after IFNG treatment (Fig. 5b, c), even though they responded to IFNG with increased synthesis of downstream effectors such as IDO1, GBP4, and CXCL11 (Fig. 5a). Although it is very little known about metabolic changes in MSC when they are exposed to inflammatory cytokines such as IFNG, and how that would corelate with their immunomodulatory capabilities, it is possible to draw a parallel with metabolic changes during macrophage or T-cell activation $^{62,63}$. Increase in both energy production and biomass such as amino acids, lipids, and nucleic acids, is necessary to provide cellular building blocks to mobilize all the resources required to fully activate and use their hostdefense mechanisms. Since such metabolic change was not detected in iMSC TEX cells, it is likely that they may not be capable of exerting expected immunomodulatory response in an inflammatory environment. Despite the similarity between iMSC ARG (T) and $\mathrm{nMSC}(\mathrm{T})$, metabolism of iMSC ARG was different to nMSC. This signifies the hypothesis that IFNG has a potent effect on stem cell physiology, leading cells to a specific metabolic state, despite the metabolic differences prior to stimulation.

The current study has several limitations, i.e., testing of immunomodulatory properties has not been done in animal model setting; nonetheless, it still demonstrates the importance of validating clinically relevant characteristics of iMSC, especially in an isogeneic system. The results of our mass spectrometry and metabolomics analyses presented provide the required evidence and the animal data would not necessarily add much more value. We have also illustrated how metabolomics can be used as a reliable tool for validating cellular response to proinflammatory stimuli. In spite of the increase in transcription of marker genes downstream of IFNG receptor activation, the signal did not reach the threshold required for setting in motion an immunomodulatory mechanism.

\footnotetext{
Acknowledgements

Dr. Klontzas and Mantalaris were funded by EU Horizon 2020 ITN SymBioSys (grant agreement 675585). We thank Dr. Yahnua Hu and Dr. Qingbo Xu from King's College London for help with teratoma assay. Animal procedures were approved by the UK Home Office (PPL70/8944). We also thank Dr. Vladimir Jovanovic from the Department of Biology, Chemistry and Pharmacy at the Freie Universität Berlin for help with statistical analyses and Dr. Morgan Stanton from VitroLabs for critical reading of the manuscript. The research was supported by the National Institute for Health Research (NIHR) Biomedical Research Centre based at Guy's and St Thomas' NHS Foundation Trust and King's College London. The views expressed are those of the authors and not necessarily those of the NHS, the NIHR or the Department of Health. We thank Dr. Yahnua Hu and Dr. Xingbo Xu from King's College London for help with teratoma assay. Animal procedures were approved by the UK Home Office (PPL70/8944). Katsumi cell line was a gift from Eric So (King's College London, UK).
}

\section{Author details}

${ }^{1}$ Department of Women and Children's Health, King's College London, Guy's Hospital, London, UK. Department of Chemical Engineering, Imperial College London, London, UK. ${ }^{3}$ Genomic Medicine, Houston Methodist Research Institute, Houston, TX, USA. ${ }^{4}$ Department of Haemato-oncology, Rayne Institute, King's College London, London, UK. ${ }^{5}$ Histology Laboratory, Wolfson Centre for Age-Related Diseases, King's College London, London, UK. 'Present address: Wallace H. Coulter Department of Biomedical Engineering, Georgia Institute of Technology, 950 Atlantic Drive, Engineering Biosciences Building, Rm 3016, Atlanta, GA 30332, USA

\section{Authors' contributions \\ L.D., M.E.K., and A.C.: conception and design, collection and/or assembly of data, data analysis and interpretation, manuscript writing; A.G. and C.H., conception and design, collection and/or assembly of data, data analysis and interpretation. F.D., A.M., Y.K., and D.I.: conception and design, data interpretation, manuscript writing, final approval of manuscript, financial support.}

\section{Conflict of interest}

The authors declare that they have no conflict of interest.

\section{Publisher's note}

Springer Nature remains neutral with regard to jurisdictional claims in published maps and institutional affiliations.

Supplementary Information accompanies this paper at (https://doi.org/ 10.1038/s41419-019-1498-0).

Received: 9 October 2018 Revised: 25 January 2019 Accepted: 25 February 2019

Published online: 20 March 2019

\section{References}

1. Haniffa, M. A., Collin, M. P., Buckley, C. D. \& Dazzi, F. Mesenchymal stem cells: the fibroblasts' new clothes? Haematologica 94, 258-263 (2009).

2. Haniffa, M. A. et al. Adult human fibroblasts are potent immunoregulatory cells and functionally equivalent to mesenchymal stem cells. J. Immunol. 179, 1595-1604 (2007).

3. Nombela-Arrieta, C., Ritz, J. \& Silberstein, L. E. The elusive nature and function of mesenchymal stem cells. Nat. Rev. Mol. Cell Biol. 12, 126-131 (2011).

4. Trento, C. et al. Manufacturing mesenchymal stromal cells for the treatment of graft-versus-host disease: a survey among centers affiliated with the European Society for Blood and Marrow Transplantation. Biol. Blood. Marrow Transplant. 24, 2365-2370 (2018).

5. Wang, Y., Chen, X., Cao, W. \& Shi, Y. Plasticity of mesenchymal stem cells in immunomodulation: pathological and therapeutic implications. Nat. Immunol. 15, 1009-1016 (2014).

6. Samsonraj, R. M. et al. Multifaceted characterization of human mesenchymal stem cells for use in regenerative medicine. Stem. Cells Transl. Med. 6, 2173-2185 (2017)

7. Galipeau, J. et al. International Society for Cellular Therapy perspective on immune functional assays for mesenchymal stromal cells as potency release criterion for advanced phase clinical trials. Cytotherapy 18, 151-159 (2016).

8. Viswanathan, S. et al. Soliciting strategies for developing cell-based reference materials to advance mesenchymal stromal cell research and clinical translation. Stem. Cells Dev. 23, 1157-1167 (2014). 2014.

9. Bianco, P. et al. The meaning, the sense and the significance: translating the science of mesenchymal stem cells into medicine. Nat. Med. 19, 35-42 (2013).

10. Hawkins, K. E. et al. Embryonic stem cell-derived mesenchymal stem cells (MSCs) Have a superior neuroprotective capacity over fetal MSCs in the hypoxic-ischemic mouse brain. Stem. Cells Transl. Med. 7, 439-449 (2018).

11. Sheyn, D. et al. Human induced pluripotent stem cells differentiate into functional mesenchymal stem cells and repair bone defects. Stem. Cells Transl. Med. 5, 1447-1460 (2016).

12. Koch, J. M., D'Souza, S. S., Schwahn, D. J., Dixon, I. \& Hacker, T. A. Mesenchymoangioblast-derived mesenchymal stromal cells inhibit cell 
damage, tissue damage and improve peripheral blood flow following hindlimb ischemic injury in mice. Cytotherapy 18, 219-228 (2016).

13. Luzzani, C. et al. A therapy-grade protocol for differentiation of pluripotent stem cells into mesenchymal stem cells using platelet lysate as supplement. Stem. Cell Res. Ther. 6, 6 (2015).

14. Zhao, Q. et al. MSCs derived from iPSCs with a modified protocol are tumortropic but have much less potential to promote tumors than bone marrow MSCs. Proc. Natl Acad. Sci. USA 112, 530-535 (2015).

15. Kimbrel, E. A. et al. Mesenchymal stem cell population derived from human pluripotent stem cells displays potent immunomodulatory and therapeutic properties. Stem. Cells Dev. 23, 1611-1624 (2014).

16. Guzzo, R. M., Gibson, J., Xu, R. H., Lee, F. Y. \& Drissi, H. Efficient differentiation of human iPSC-derived mesenchymal stem cells to chondroprogenitor cells. J. Cell Biochem. 114, 480-490 (2013).

17. Vodyanik, M. A. et al. A mesoderm-derived precursor for mesenchymal stem and endothelial cells. Cell Stem. Cell 7, 718-729 (2010).

18. Boyd, N. L., Robbins, K. R., Dhara, S. K., West, F. D. \& Stice, S. L. Human embryonic stem cell-derived mesoderm-like epithelium transitions to mesenchymal progenitor cells. Tissue Eng. Part. A. 15, 1897-1907 (2009).

19. Karlsson, C. et al. Human embryonic stem cell-derived mesenchymal progenitors - potential in regenerative medicine. Stem. Cell Res. 3, 39-50 (2009).

20. Lian, Q. et al. Derivation of clinically compliant MSCs from CD105 + , CD24differentiated human ESCs. Stem. Cells 25, 425-436 (2007).

21. Barberi, T., Willis, L. M., Socci, N. D. \& Studer, L. Derivation of multipotent mesenchymal precursors from human embryonic stem cells. PLoS. Med. 2, e161 (2005).

22. King, D. First human allogeneic clinical trial commences iPSC-derived mesenchymal stem cells. 2017. https://cellculturedish.com/2017/07/firsthuman-allogeneic-clinical-trial-commences-ipsc-derived-mesenchymal-stemcells/ Accessed 19 Aug 2018

23. Clinicaltrials.gov https://clinicaltrials.gov/ct2/show/NCT02923375 Accessed 19 Aug 2018.

24. Frobel, J. et al. Epigenetic rejuvenation of mesenchymal stromal cells derived from induced pluripotent stem cells. Stem Cell Rep. 3, 414-422 (2014).

25. Devito, L. et al. Wharton's jelly mesenchymal stromal/stem cells derived under chemically defined animal product-free low oxygen conditions are rich in MSCA-1 $(+)$ subpopulation. Regen. Med. 9, 723-732 (2014).

26. Miere, C., Devito, L. \& Ilic, D. Sendai virus-based reprogramming of mesenchymal stromal/stem cells from umbilical cord wharton's jelly into induced pluripotent stem cells. Methods Mol. Biol. 1357, 33-44 (2016).

27. Devito, L. et al. Induced pluripotent stem cell line from an atopic dermatitis patient heterozygous for c.2282del4 mutation in filaggrin: KCLi001-A. Stem. Cell Res. 31, 122-126 (2018).

28. Petrova, A. et al. 3D In vitro model of a functional epidermal permeability barrier from human embryonic stem cells and induced pluripotent stem cells. Stem. Cell Rep. 2, 675-689 (2014).

29. Badraiq, H. et al. Effects of maternal obesity on Wharton's Jelly mesenchymal stromal cells. Sci. Rep. 7, 17595 (2017).

30. Shao, K. et al. Induced pluripotent mesenchymal stromal cell clones retain donor-derived differences in DNA methylation profiles. Mol. Ther. 21, 240-250 (2013).

31. Klontzas, M. E., Vernardis, S. I., Heliotis, M., Tsiridis, E. \& Mantalaris, A. Metabolomics analysis of the osteogenic differentiation of umbilical cord blood mesenchymal stem cells reveals differential sensitivity to osteogenic agents. Stem. Cells Dev. 26, 723-733 (2017).

32. Vernardis, S. I., Terzoudis, K., Panoskaltsis, N. \& Mantalaris, A. Human embryonic and induced pluripotent stem cells maintain phenotype but alter their metabolism after exposure to ROCK inhibitor. Sci. Rep. 7, 42138 (2017).

33. Vernardis, S. I., Goudar, C. T. \& Klapa, M. I. Metabolic profiling reveals that time related physiological changes in mammalian cell perfusion cultures are bioreactor scale independent. Metab. Eng. 19, 1-9 (2013).

34. Silva, L. P. et al. Measurement of DNA concentration as a normalization strategy for metabolomic data from adherent cell lines. Anal. Chem. 85, 9536-9542 (2013)

35. Kanani, H., Chrysanthopoulos, P. K. \& Klapa, M. I. Standardizing GC-MS metabolomics. J. Chromatogr. B. Anal. Technol. Biomed. Life. Sci. 871, 191-201 (2008).

36. Kanani, H. H. \& Klapa, M. I. Data correction strategy for metabolomics analysis using gas chromatography-mass spectrometry. Metab. Eng. 9, 39-51 (2007).

37. Tusher, V. G., Tibshirani, R. \& Chu, G. Significance analysis of microarrays applied to the ionizing radiation response. Proc. Natl Acad. Sci. USA 98, 5116-5121 (2001).
38. Sobiesiak, M. et al. The mesenchymal stem cell antigen MSCA-1 is identical to tissue non-specific alkaline phosphatase. Stem. Cells Dev. 19 669-677 (2010).

39. Bühring, $\mathrm{H}$. J. et al. Phenotypic characterization of distinct human bone marrow-derived MSC subsets. Ann. N. Y. Acad. Sci. 1176, 124-134 (2019).

40. Gronthos, S. et al. A novel monoclonal antibody (STRO-3) identifies an isoform of tissue nonspecific alkaline phosphatase expressed by multipotent bone marrow stromal stem cells. Stem. Cells Dev. 16, 953-963 (2007).

41. Stephenson, E. et al. Derivation and propagation of human embryonic stem cell lines from frozen embryos in an animal product-free environment. Nat. Protoc. 7, 1366-1381 (2012).

42. Ma, S., et al. Immunobiology of mesenchymal stem cells. Cell Death Differ. 21, 216-225.

43. Marigo, I. \& Dazzi, F. The immunomodulatory properties of mesenchymal stem cells. Semin. Immunopathol. 33, 593-602 (2011).

44. Krampera, M. et al. Role for interferon-gamma in the immunomodulatory activity of human bone marrow mesenchymal stem cells. Stem. Cells 24, 386-398 (2006).

45. Vestal, D. J. The guanylate-binding proteins (GBPs): proinflammatory cytokineinduced members of the dynamin superfamily with unique GTPase activity. J. Interferon Cytokine Res. 25, 435-443 (2005).

46. Tensen, C. P. et al. Genomic organization, sequence and transcriptional regulation of the human CXCL 11(1) gene. Biochim. Biophys. Acta 1446, 167-172 (1999).

47. Sakaki-Yumoto, M., Katsuno, Y. \& Derynck, R. TGF- $\beta$ family signaling in stem cells. Biochim. Biophys. Acta 1830, 2280-2296 (2013).

48. Huang, Y. C., Parolini, O., La Rocca, G. \& Deng, L. Umbilical cord versus bone marrow derived mesenchymal stromal cells. Stem. Cells Dev. 21, 2900-2903 (2012).

49. De Kock, J. et al. Mesoderm-derived stem cells: the link between the transcriptome and their differentiation potential. Stem. Cells Dev. 21, 3309-3323 (2012).

50. Corrao, S. et al. Umbilical cord revisited: from Wharton's jelly myofibroblasts to mesenchymal stem cells. Histol. Histopathol. 28, 1235-1244 (2013).

51. Eliasson, P. \& Jonsson, J. I. The hematopoietic stem cell niche: low in oxygen but a nice place to be. J. Cell Physiol. 222, 17-22 (2010).

52. Yang, S. et al. Defined xenogeneic-free and hypoxic environment provides superior conditions for long-term expansion of human adipose-derived stem cells. Tissue Eng. Part. C. Methods 18, 593-602 (2012).

53. Drela, K. et al. Low oxygen atmosphere facilitates proliferation and maintains undifferentiated state of umbilical cord mesenchymal stem cells in an hypoxia inducible factor-dependent manner. Cytotherapy 16, 881-892 (2014).

54. López, L., Seshareddy, K., Trevino, E., Cox, J. \& Weiss, M. L. Evaluating the impact of oxygen concentration and plating density on human Wharton's jellyderived mesenchymal stromal cells. Open Tissue Eng. Regen. Med. J. 4, 82-94 (2011).

55. Lavrentieva, A., Majore, I., Kasper, C. \& Hass, R. Effects of hypoxic culture conditions on umbilical cordderived human mesenchymal stem cells. Cell Commun. Signal. 16, 18 (2010).

56. Nekanti, U., Dastidar, S., Venugopal, P., Totey, S. \& Ta, M. Increased proliferation and analysis of differential gene expression in human Wharton's jelly-derived mesenchymal stromal cells under hypoxia. Int. J. Biol. Sci. 6, 499-512 (2010).

57. Brewer, G. J., Torricelli, J. R., Evege, E. K. \& Price, P. J. Optimized survival of hippocampal neurons in B27-supplemented Neurobasal, a new serum-free medium combination. J. Neurosci. Res. 35, 567-576 (1993).

58. Brewer, G. J. \& Cotman, C. W. Survival and growth of hippocampal neurons in defined medium at low density: advantages of a sandwich culture technique or low oxygen. Brain Res. 494, 65-74 (1989).

59. Carcamo-Orive, I. et al. Analysis of transcriptional variability in a large human iPSC library reveals genetic and non-genetic determinants of heterogeneity. Cell Stem. Cell 20, 518-532 (2017).

60. DeBoever, $C$. et al. Large-scale profiling reveals the influence of genetic variation on gene expression in human induced pluripotent stem cells. Cell Stem. Cell 20, 533-546 (2017).

61. Kilpinen, $\mathrm{H}$. et al. Common genetic variation drives molecular heterogeneity in human iPSCs. Nature 546, 370-375 (2017).

62. Domblides, C., Lartigue, L. \& Faustin, B. Metabolic stress in the immune function of T cells, macrophages and dendritic cells. Cells 7, E68 (2018).

63. Zhu, L., Zhao, Q., Yang, T., Ding, W. \& Zhao, Y. Cellular metabolism and macrophage functional polarization. Int. Rev. Immunol. 34, 82-100 (2015). 